\title{
Particle-particle coating in a cyclomix impact mixer
}

\author{
Y. Ouabbas, J. Dodds *, L. Galet, A. Chamayou, M. Baron \\ Centre RAPSODEE UMR CNRS 2392, Ecole des Mines d'Albi-Carmaux, Campus Jarlard - 80013 Albi, France
}

A B S T R A C T

Dry particle coating can be used to create new-generation materials by combining different powders exhibiting different physical and/or chemical properties. In such processes relatively large particles (host particles) are mechanically coated with fine particles (guest particles), without using solvents and subsequent drying, to create new functionalities or to improve initial characteristics. The coating of a host powder by guest particles can be performed in many different ways ranging from simple stirring of two components, to high energy impact coating.

The purpose here is to examine the use of a Cyclomix high shear mixer granulator for the surface modification of silica particles by dry coating with magnesium stearate, and of cornstarch dry coated with hydrophilic or hydrophobic fume silica. Several powder characterisation methods have been used to study the physicochemical properties of the coated particles. These include: Observation by environmental scanning electron microscopy (ESEM), Characterization of cohesion and flowability by tap density measurements, Determination of wettability by measurements of the contact angle between the coated particles and water. Measurement of moisture adsorption-desorption isotherms of uncoated and coated particles, and Tribo-electrification characteristics of the powders. The results show that the surface properties of the two host particles are changed, and that the Cyclomix can be used for dry particle coating to modify the properties of silica gel and cornstarch powders.

\author{
Keywords: \\ Dry coating \\ Starch \\ Silica gel \\ Magnesium stearate \\ Fume silica \\ Cyclomix \\ Surface morphology \\ Moisture adsorption \\ Flowability \\ Tribo-charging
}

\section{Introduction}

Dry particle coating can find application in many industries for changing the surface properties and/or functionality of powders. For example it may be used in the pharmaceutical, cosmetic, food or ceramics industries for modification of flowability, wettability (hydrophobic/hydrophilic properties), dispersability and rate of dissolution, flavour, particle shape, electrostatic, optical, electric, magnetic, particle properties. As indicated in Fig. 1, in such coating processes, powders with relatively large particle sizes (host particles: 1-500 $\mu \mathrm{m}$ ) are mechanically coated with fine particles (guest particles: $0.1-50 \mu \mathrm{m}$ ) in order to create a new functionality or to improve their initial characteristics [1]. The success of the process depends on the "affinity" of the guest particle for the host particles and the mechanical forces brought to bring them together. Since the guest particles are very small, Van der Waals interactions are strong enough to keep them firmly attached to the large host particles [2]. Thus, either a discrete or continuous coating of guest particles can be achieved depending on the operating conditions such as mechanical force used, processing time, weight fraction of guest to host particles and particle properties [3]. Multiple layering is possible when using different coating materials and processing them

\footnotetext{
* Corresponding author.

E-mail address: john.dodds@enstimac.fr (J. Dodds).
}

one after other. Kangwantrakol et al. (2001) [4] have described a method to make multilayer coating of guest particles.

Dry particle coating is very closely related to ordered mixing (a term coined by Hersey, 1975), where a mixture is formed by covering the surface of larger particles with smaller particles. However in dry particle coating, the aim is to make the surface covering more permanent with stronger physical (or chemical) bonding [3]. Initial work on ordered mixing was done by Hersey [5]. The concept of ordered mixing was also taken one step further (to dry coating) by using dry impact blending, as described in a series of papers by a Japanese group [6-9]. Several different machines have been developed for dry coating allowing the creation of new types of materials. Most of the devices which have been used for dry powder coating are rather specialised laboratory type equipment and not readily available for processing amounts greater than several grams of powder. Pfeffer [3] gives descriptions of these different dry coating systems. Mujumbar et al. [10] have studied dry coating to enhance the moisture resistance of ground magnesium powder by coating its surface with carnuba wax using Magnetically Assisted Impaction Coating (MAIC) and two high-speed impaction devices, the Hybridizer and Mechanofusion device. Yang et al. [11] showed that it is possible to improve the flowability of cornstarch by coating with nanosized silica, using dry coating devices such as MAIC and Hybridizer.

Here we describe an experimental investigation of dry powder coating using a "Cyclomix" high shear mixer. This apparatus is a vertical cone shaped high shear mixer made by Hosokawa Micron and is 


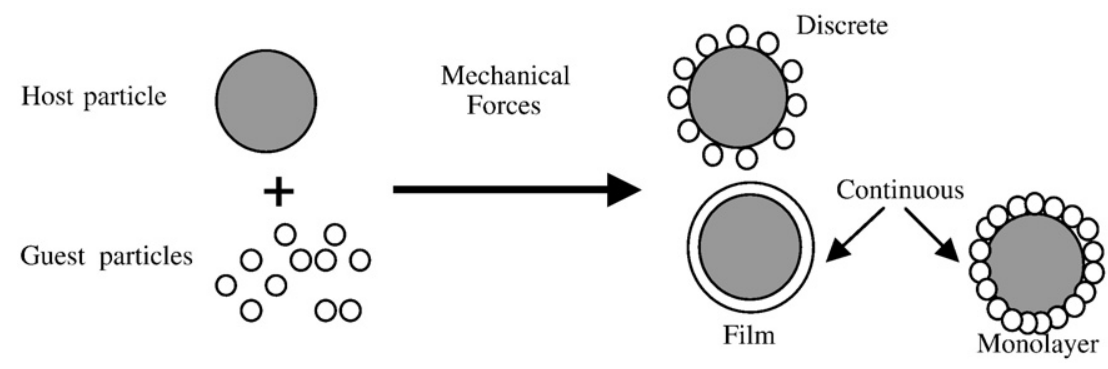

Fig. 1. Dry powder coating.

available in a range of sizes from $1 \mathrm{~L}$ to $500 \mathrm{~L}$. $\mathrm{Ng}$ et al. [12] presented an experimental study on the solids motion in a Cyclomix by the positron emission particle tracking technique. Kwan et al [13] studied the granulation of hollow glass beads in a range of sizes of Cyclomix for scaleup. Here we report experiments which have been performed using a Cyclomix for mechanical dry coating of silica gel particles with magnesium stearate, and for coating starch particles with hydrophilic and hydrophobic fume silica. The effects of these surface coatings on the flowability, the wettability and tribo-charging are determined.

In a previous paper on dry particle coating [14] we presented an experimental technique based on dry powder particle size analysis for determining the force required to remove the coating layer. This allowed us to compare the mechanical resistance of coatings made using different techniques such as manual shaking, a Turbula mixer, and a Hybridizer. The advantage of the Cyclomix is that it can treat large quantities of powder and is available for industrial processing, and one of the aims of this paper is to evaluate the resistance of a Cyclomix coating with that made by a Hybridizer.

\section{Experimental}

\subsection{Powders}

Silica gel powder supplied by Merck and usually used for chromatography column has been chosen as host particles for dry coating. This silica gel has a hydrophilic porous structure which readily absorbs moisture and as shown in Fig. 3(a) the particles are irregularly shaped and their surface is rough. Hydrophobic magnesium stearate (MgSt) supplied by Chimiray is used as guest particles. The MgSt is a fine, white, greasy and cohesive powder widely used in pharmaceutical formulation as a lubricant. Observation by ESEM showed a wide distribution of size and shape including needle and plate like particles. The starch was Corn Starch B from Roquette Freres. The fumed silica was from Degussa; Aerosil R200 hydrophilic, Aerosil R974 hydrophobic. The properties of host and guest particles used in the experiments are summarized in Table 1.

Table 1

Properties of host and guest particles

\begin{tabular}{|c|c|c|c|c|}
\hline Particles & $\begin{array}{l}\text { Size } d_{50} \mu \mathrm{m} \\
\text { mastersizer }\end{array}$ & $\begin{array}{l}\text { Solid density } \mathrm{g} / \mathrm{cm}^{3} \\
\text { HeliumPycnometer }\end{array}$ & $\begin{array}{l}\text { Wetting } \\
\text { characteristics }\end{array}$ & $\begin{array}{l}\text { Specific surface } \\
\left(\mathrm{m}^{2} / \mathrm{g}\right) \\
(\text { ASAP 2010) }\end{array}$ \\
\hline Silica gel & 55 & 2.07 & Hydrophilic & 510 \\
\hline Cornstarch & 13 & 1.47 & Hydrophilic & - \\
\hline $\begin{array}{r}\text { Aerosil } \\
\text { R200 }\end{array}$ & 0.12 & 2.27 & Hydrophilic & 200 \\
\hline $\begin{array}{r}\text { Aerosil } \\
\text { R974 }\end{array}$ & 0.12 & 2.27 & Hydrophobic & 174 \\
\hline MgSt & 4.6 & 1.04 & Hydrophobic & 6 \\
\hline
\end{tabular}

\subsection{Coating processes}

The mass percentage of guest particles required in a coating experiment is calculated based on the assumption of $100 \%$ surface coverage of the host particles with a monolayer of guest particles. It is assumed that both host and guest particles are spherical (respective sizes $D_{\text {host }}$ $D_{\text {guest }}$, respective densities $\rho_{\text {host }} \rho_{\text {guest }}$ ), and do not change shape during the coating process. Based on these assumptions, the mass percentage $(W)$ of guest particles for $100 \%$ coverage can be written as [10]:

$W(\%)=\frac{\left(N D_{\text {guest }}^{3} \rho_{\text {guest }}\right)}{\left(D_{\text {host }}^{3} \rho_{\text {host }}\right)+\left(N D_{\text {guest }}^{3} \rho_{\text {guest }}\right)} \times 100$

For $D_{\text {host }} \gg D_{\text {guest }}$ (here, $D_{\text {host }} / D_{\text {guest }} \approx 10$ ), the number $N$ of guest particles per host particle is given by the expression:

$N=\frac{4\left(D_{\text {host }}+D_{\text {guest }}\right)^{2}}{D_{\text {guest }}^{2}}$

From Eq. (1), the percentage of guest particles needed to coat silica gel particles is determined to be $15 \%$. In addition, coating experiments have been carried out with $1 \%$ and $5 \%$ of magnesium stearate in order to compare what happens mass ratios of guest to host particles lower than $15 \%$. The percentage of fume silica guest particles needed to coat starch particles is determined to be $1 \%$.

A 1 L capacity Cyclomix (Hosokawa Micron B.V.) high impact mixer is used for the coating experiments. The mixer has four pairs of flat-bladed impellers located in series on a centrally located high-speed top driven rotating shaft. This eliminates seals and bearings from the product zone. The shaft is fitted with paddle-shaped mixing elements, which rotate close to the inner vessel wall [12]. Fig. 2 shows a schematic diagram of the action of a Cyclomix mixer. The working principle of the Cyclomix differs from existing mixing techniques and from other devices used for dry

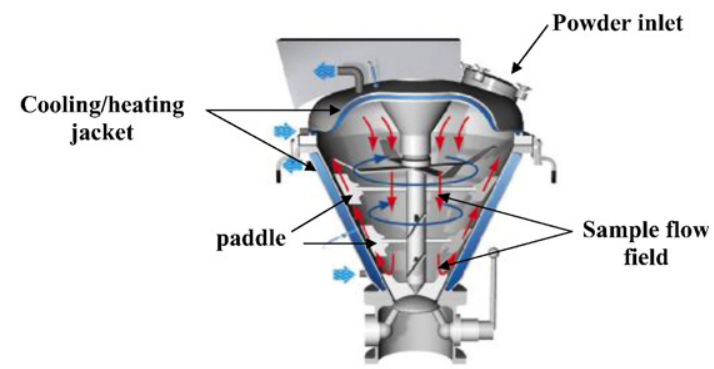

Fig. 2. Schematic diagram of a Cyclomix high shear mixer. 
powder coating by the specific interaction between mixing element and vessel wall. The powder (host and guest particles) is loaded into the conical mixing vessel from the top; the degree of filling can range between 30 and $100 \%$. The high-speed of rotation (up to $2500 \mathrm{rpm}$ ) of the paddles and the conical shape of the vessel force the product from the bottom to the upper zone of the vessel. Upon reaching the top, the product flows downwards into the centre of the vessel. This flow pattern results in fast macromixing. During the upward motion, the particles are accelerated by the paddles and intensively mixed by friction with vessel walls. The operating conditions used in all experiments presented here are $80 \%$ filling and $1500 \mathrm{rpm}$ for $5 \mathrm{~min}$.

\section{Characterization}

The uncoated and coated particles were examined by means of an environmental scanning electron microscopy (ESEM) to study the surface morphology and particle shapes before and after coating. A Volumeter (or Tap-tap) tapped density tester was used to examine the flowability of the uncoated and coated samples. The tapped density was obtained by taking volume readings of a known weight ( $\mathrm{m}$ ) of powder sample contained in a measuring cylinder and subjected mechanical tapping. To reduce variability due the action of filling the cylinder the "initial" volume is taken as that after $10 \operatorname{taps}\left(V_{10}\right)$ the final volume is that when no change in volume takes place $\left(V_{\infty}\right)$. The flow properties and cohesion of the uncoated and coated powders were evaluated by the Carr's flowability index (IC) and Hausner's ratio (HR).

$\rho_{0}=m / V_{10} \quad \rho_{\infty}=m / V_{\infty}$

Carr index $\mathrm{IC}=\frac{\rho_{\infty}-\rho_{0}}{\rho_{\infty}} \%$

Hausner ratio $\mathrm{HR}=\frac{\rho_{\infty}}{\rho_{0}}$

Flowability is classed as a function of IC as follows:

$<15 \%$ good 15 to $25 \%$ poor $>25 \%$ bad

Cohesivity is classed as a function of HR as follows:

1.0 to 1.2 low cohesion, 1.2 to 1.4 medium cohesion, $>1.4$ high cohesion.

The wettability was studied by the sessile drop method using an ILMS instrument (GBX S.A.) [15,16]. A small water drop of $10 \mu \mathrm{l}$ is deposited on the surface of a powder bed prepared for each sample. The shape of the drop profile is observed and used to determine the contact angle.

The moisture adsorption-desorption isotherms of uncoated and coated particles were obtained by using a Dynamic Vapour Sorption (DVS) analyzer (Surface Measurement Systems Ltd. UK).

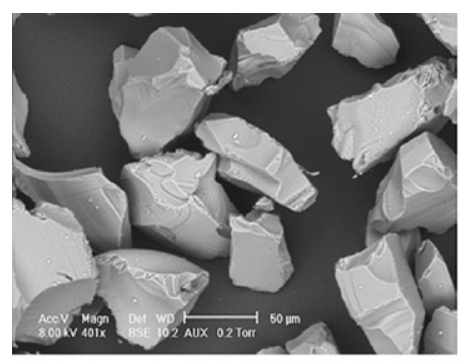

(a) Silica gel as received

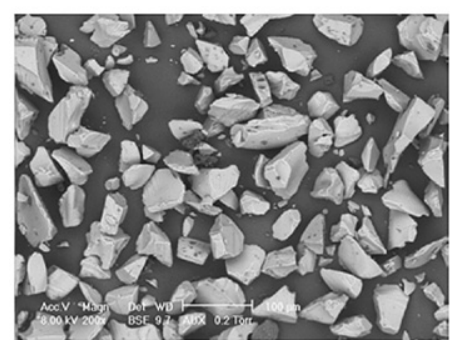

(d) Silica $+1 \%$ MgSt Cyclomix

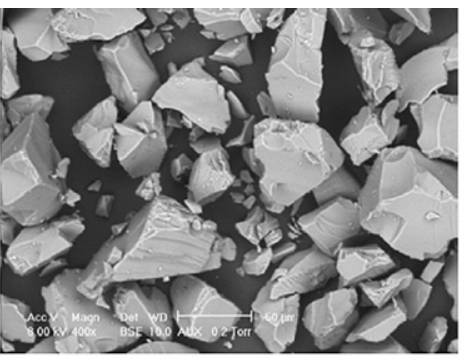

(b) Silica gel after treatment alone in the Cyclomix

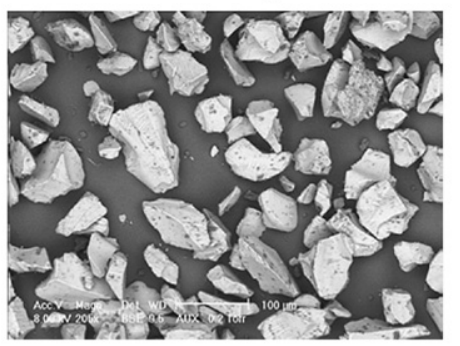

(e) Silica $+5 \% \mathrm{MgSt}$ Cyclomix

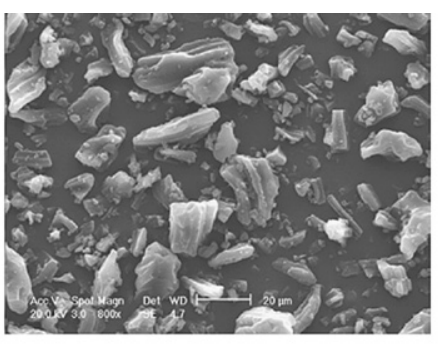

(c) Magnesium Stearate $(\mathrm{MgSt})$

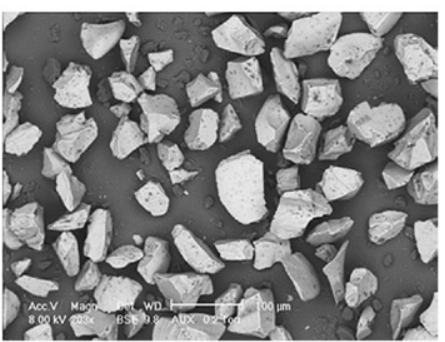

(f) Silica $+15 \%$ MgSt Cyclomix

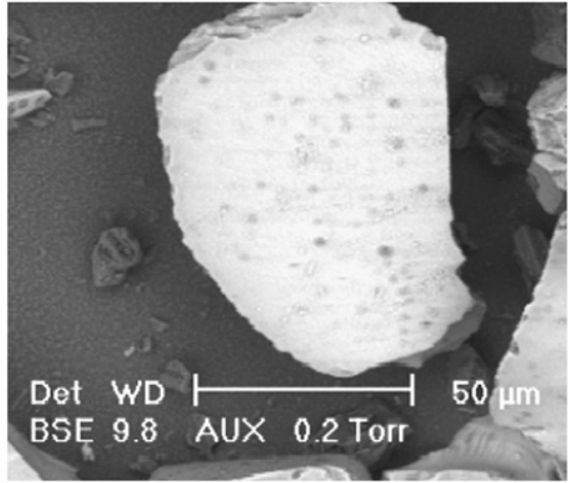

Enlargement of the central particle in figure 3(f)

Fig. 3. ESEM photos of the silica gel magnesium stearate system. 


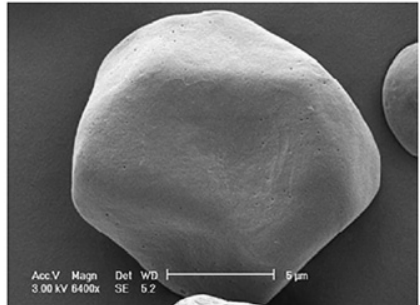

(a) Starch particle as received

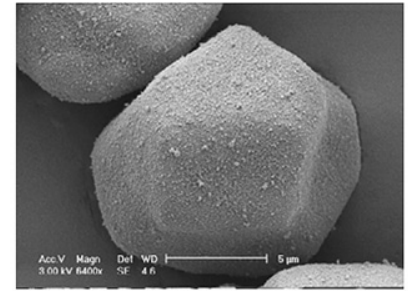

(c) Starch particle with 1\% Aerosil R200

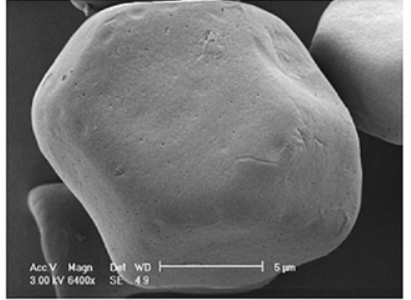

(b) Starch particle treated in Cyclomix

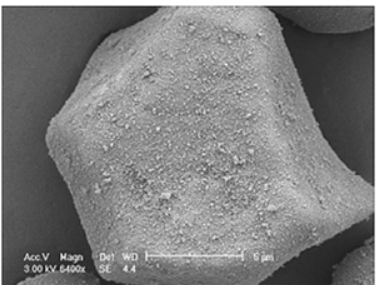

(d) Starch particle with $1 \%$ Aerosil R974

Fig. 4. ESEM photos of starch particles Fumed silca system.

Tribo-electric charging experiments were made with approximately $8 \mathrm{~g}$ samples of the powders rotated in a $250 \mathrm{ml}$ stainless steel beaker held at an angle of $45^{\circ}$, and then pouring the powder into a Valitec Faraday cage connected to a Keithley 6514 electrometer [17].

\section{Results and discussion}

\subsection{Surface morphology}

\subsubsection{Silica gel magnesium stearate experiments}

As shown on Fig. 3(a), the silica gel before treatment has irregularly shaped particles. After processing in the Cyclomix, the mean size of the silica gel particles was found to be slightly smaller probably because of some grinding action by the high shear in the mixer (Fig. 3(b)). The magnesium stearate Fig. 3(c) has a wide size distribution and a large variety of particle shapes including needles and platelets. The surface morphology of particles coated with $1 \%, 5 \%$ and $15 \%(w / w)$ of $M g S t$ in the Cyclomix is shown in Fig. 3 ( $\mathrm{d}$ to $\mathrm{f}$ ). The guest particles are individually distributed on the surface of silica gel particles (see Fig. 3(f) and the enlargement in Fig. 3(g)). The coverage increases as the percentage of $\mathrm{MgSt}$ is increased from $1 \%$ to $5 \%$ to $15 \% \mathrm{w} / \mathrm{w}$. Coating seems to be more uniform for the particles coated with $15 \%$ of MgSt which seems to be more smeared over the surface.

\subsubsection{Starch fumed silica experiments}

Fig. 4( $\mathrm{a}$ and $\mathrm{b})$ shows that the starch particles are rounded and smooth. There was no significant breakage due to processing in the Cyclomix. The coating with fumed silica is seen (Fig. 6 a and b) to be as individual particles set in the surface.

\subsection{Coating resistance tests}

The resistance of the coating was determined using the technique based on dry powder particle size analysis technique presented elsewhere [14]. Figs. 5 and 6 show the particle size distributions of the silica MgSt systems obtained with a Malvern Mastersizer dry feed system with low 0.5 bar (Fig. 5) and high 3.5 (Fig. 6) dispersing pressure. The volume distributions show very little difference. The number distributions show that at 3.5 bars pressure there is a small release of fine MgSt particles but that the coating is substantially still in place. Similar results were obtained for the Starch Fume silica system.
These results, and comparison with results presented elsewhere [14] for the Nara Hybridiser, indicate that the Cyclomix does give mechanically resistant coatings with these two sets of powders. The number distribution curve for the $15 \% \mathrm{MgSt}$ coating at 3.5 bar dispersing pressure (Fig. 6(b)) indicates a certain degree of deagglomeration, but the fact that the curve does not fully correspond

(a) Volume distribution

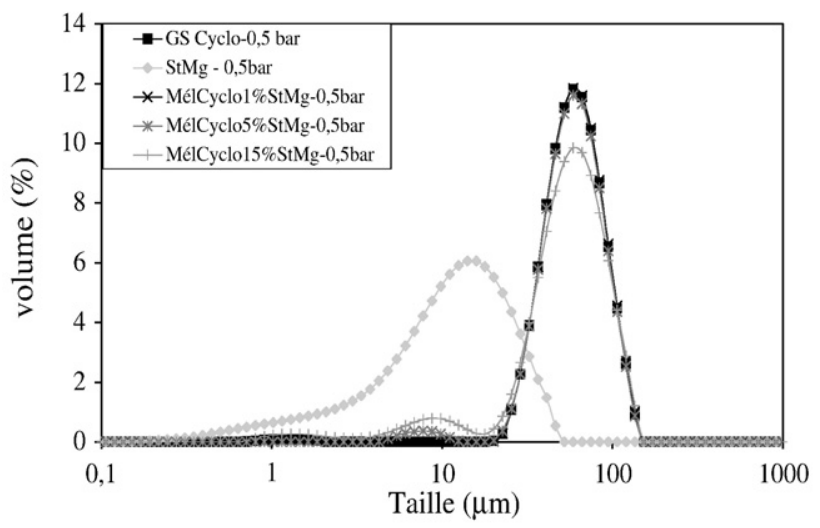

(b) Number distribution

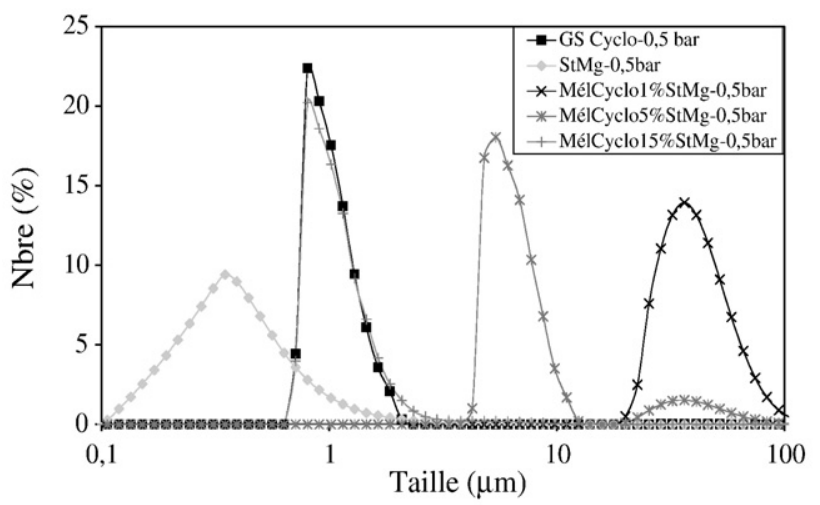

Fig. 5. Particle size distributions silica + MgSt at 0.5 bar dispersing pressure. 
(a) Volume distribution

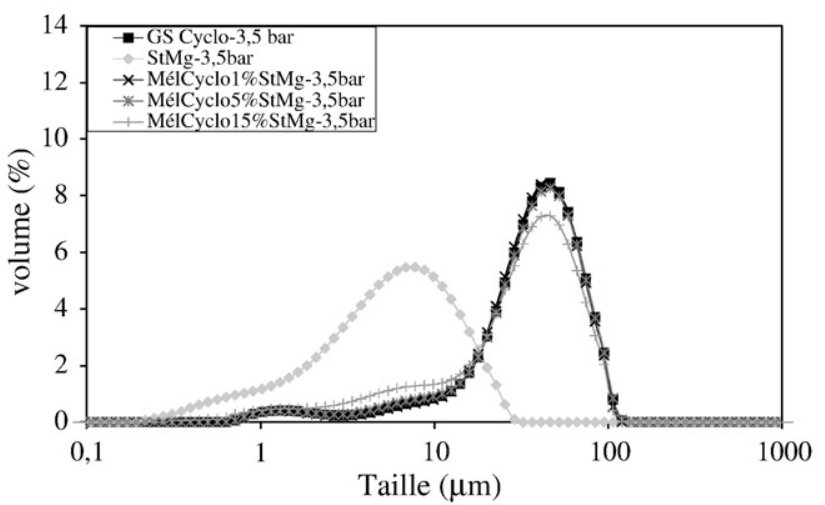

(b) Number distribution

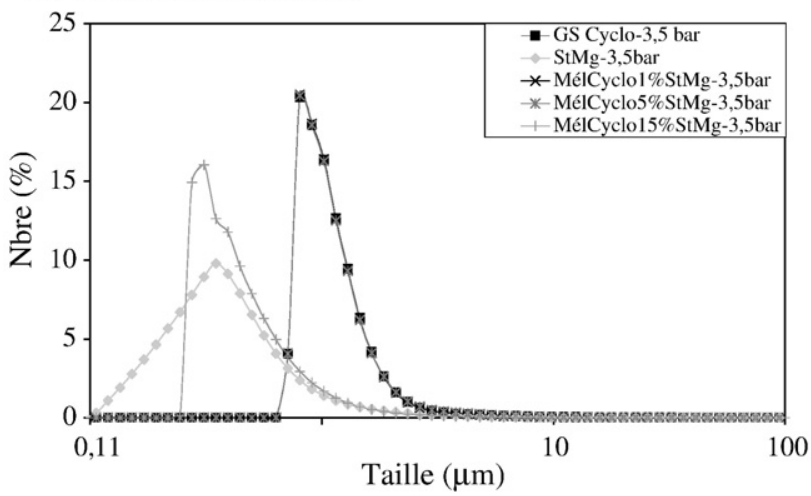

Fig. 6. Particle size distributions silica + MgSt at 3.5 bar dispersing pressure.

to that of free MgSt indicates there is not full liberation of the fine silica particles.

\subsection{Flowability tests}

\subsubsection{Silica gel and magnesium stearate}

The values of the Carr's flowability index (IC) and Hausner's ratio (HR) of uncoated and coated silica gel particles and MgSt as determined from tap-tap settling tests are given in Table 2 . The as-received silica gel powder presents good flow properties ( $I C=15.8$; $H R=1.25$ ). This is slightly changed by processing with the Cyclomix probably due to slight breakage. The MgSt powder has bad flow properties because of its high cohesivity (IC=34.1; HR=1.5). The coated particles have characteristics intermediate between those of the initial powders, i.e. poor to bad flowability.

\subsubsection{Starch and fume silica}

Fig. 7 shows the variation of the tapped density measured for the starch coated with $1 \%$, of both types of Silica in the Cyclomix. The

Table 2

Carr index (IC) and Hausner ratio (HR) Silica gel MgSt system

\begin{tabular}{lllllll}
\hline Samples & $\rho_{0} \mathrm{~g} / \mathrm{ml}$ & $\rho_{\infty} \mathrm{g} / \mathrm{ml}$ & IC & HR & Cohesion & Flowability \\
\hline Silica gel (SG) & 0.465 & 0.579 & 15.8 & 1.25 & Low & Good \\
Cyclomix SG & 0.478 & 0.568 & 19.7 & 1.19 & Low & Poor \\
SG+1\%MgSt & 0.456 & 0.564 & 19.1 & 1.24 & Low & Poor \\
SG+5\%MgSt & 0.426 & 0.560 & 23.9 & 1.31 & Medium & Bad \\
SG+15\%MgSt & 0.406 & 0.533 & 23.8 & 1.31 & Medium & Bad \\
MgSt & 0.296 & 0.450 & 34.1 & 1.52 & High & Bad \\
\hline
\end{tabular}

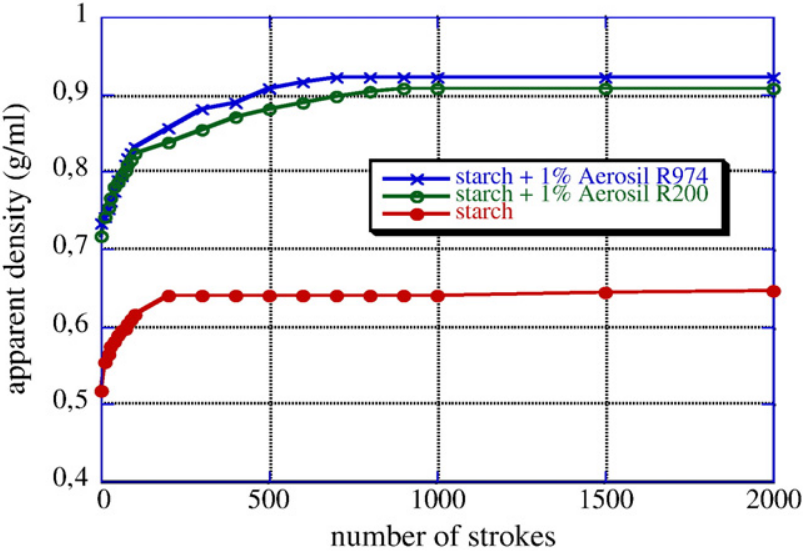

Fig. 7. Tap density kinetics for binary mixtures of starch fume silica.

results are compared to those obtained for untreated starch particles. It can be seen that the particles treated with Aerosil have higher bulk densities $(0.91$ to $0.92 \mathrm{~g} / \mathrm{ml})$ than the starch alone $(0.65 \mathrm{~g} / \mathrm{ml})$ indicating that the silica coating improves packing. Furthermore it can be seen that the settling for the starch alone is essentially ended after 300 taps whereas the curves for the treated samples requires up to 1000 taps. The Carr and Hausner indices indicate that the silica coating brings only slight changes but that the hydrophobic silica has a slightly greater effect (Table 3).

\subsection{Wettability tests}

\subsubsection{Silica gel and magnesium stearate}

Since MgSt is hydrophobic, its use as a coating should make the hydrophilic silica gel surface become hydrophobic. To evaluate the impact of coating on the reduction of the high affinity between silica gel and water, wettability tests were carried out for the uncoated particles and the silica gel particles coated in the Cyclomix. The values of the measured contact angle (at $t=0 \mathrm{~s}$ ) and the work of adhesion $\left(W_{\text {adh }}\right)$ describing the interaction between two different phases (the required work to extract the water drop from the surface of powder bed) are summarized in Table 4 .

Fig. 8 shows photos taken after two different times of a water drop placed on the uncoated silica gel powder bed. It can be seen that the water drop disappears instantaneously (after 1s) into the powder because of the high affinity between silica gel and water.

As for silica gel powder, the water drop disappears rapidly from the surface of powder bed prepared for particles coated with $1 \%$ of MgSt (after only $3 \mathrm{~s}$; Fig. 8(a)) whereas the contact angle increased and the work of adhesion decreased. The mass ratio of $1 \%$ of MgSt gives a very poor coating of the host particles and is insufficient to reduce the high affinity between silica gel and water. After coating with $5 \%$ and $15 \%$ of MgSt (Fig. 8(b), (c)), the water drop is not absorbed and remains on the surface of silica gel powder. The measured contact angle increases when the MgSt percentage increases whereas the work of adhesion decreases (Table 4). These results clearly prove that, on one hand the surface of the silica gel particles is coated by the MgSt particles and on

Table 3

Carr's index (IC) and Hausner's ratio (HR) for the Starch fume silica system

\begin{tabular}{lllllll}
\hline Sample & $\rho_{0} \mathrm{~g} / \mathrm{ml}$ & $\rho_{\infty} \mathrm{g} / \mathrm{ml}$ & IC & HR & Cohesion & Flowability \\
\hline Starch & 0.555 & 0.647 & 14.2 & 1.17 & Low & Good \\
Starch +1\% aerosil R200 & 0.741 & 0.907 & 18.3 & 1.22 & Medium & Poor \\
Starch+1\% aerosil R974 & 0.742 & 0.921 & 19.4 & 1.24 & Medium & Poor \\
\hline
\end{tabular}




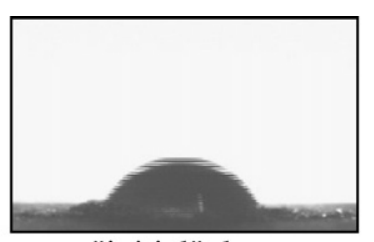

"initial" drop

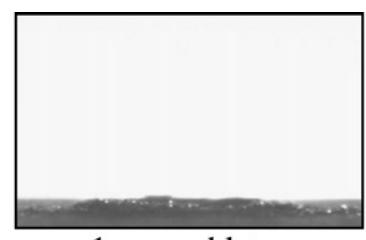

1 second later

Wettability of uncoated silica gel particles

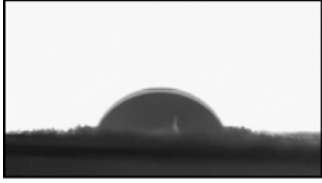

0 seconds

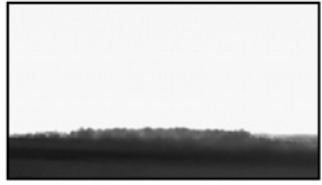

3 seconds

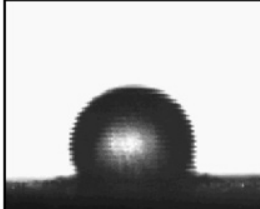

0 seconds

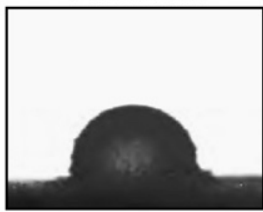

60 seconds

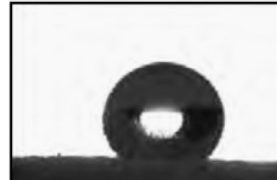

0 seconds

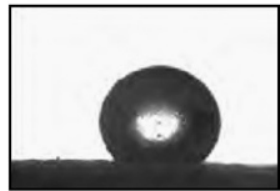

60 seconds

Wettability of coated silica particles
(a) $1 \% \mathrm{MgSt}$
(b) $5 \% \mathrm{MgSt}$
(c) $15 \% \mathrm{MgSt}$

Fig. 8. Wettability of coated silica particles.

the other hand that the affinity of the silica gel powder for water is reduced by dry coating in the Cyclomix.

\subsubsection{Starch fumed silica system}

Starch system is too hydrophilic for the sessile drop test to be applied. The liquid is immediately taken up by the starch and the drop does not remain on the powder surface. Coating with fume silica does not change this situation.

\subsection{Water vapour sorption and desorption isotherms}

\subsubsection{Silica gel magnesium stearate system}

Moisture sorption isotherms were obtained by measurement of moisture uptake using a DVS apparatus. Prior to being exposed to water vapour, the samples $(100 \mathrm{mg})$ are equilibrated at $0 \%$ relative humidity (RH) to establish a dry mass baseline. Experiments were performed with samples of untreated silica gel and silica gel coated with MgSt. The kinetics of adsorption was determined by following moisture uptake from $0 \%$ to $60 \%$ relative humidity with time. This is shown in Fig. 9. Equilibrium adsorption isotherms were then measured at $25{ }^{\circ} \mathrm{C}$ from $0 \%$ to $90 \% \mathrm{RH}$ in increments of $10 \% \mathrm{RH}$. These are shown on Fig. 10. It can be seen in Fig. 10 that the kinetics of moisture uptake is slowed by the presence of the $5 \%$ and $15 \%$ MgSt coating. In addition the final plateau reached shows that amount of moisture taken up is also changed. This equilibrium effect is found on Fig. 10 of the overall moisture adsorption isotherm. The 1\% MgSt coating has little effect as it can be seen that the kinetics curve and the

Table 4

Results of the measured contact angles and the work of adhesion

\begin{tabular}{lcc}
\hline Samples & Average contact angle $\left(\theta^{\circ}\right)$ & $W_{\text {adh }}\left(\mathrm{mN} \mathrm{m}^{-1}\right)$ \\
\hline Silica gel & 15.2 & 112 \\
$1 \%$ MgSt Cyclomix & 80 & 86 \\
$5 \%$ MgSt Cyclomix & 98 & 62 \\
$15 \%$ MgSt Cyclomix & 127 & 29 \\
MgSt & 125 & 32 \\
\hline
\end{tabular}

adsorption isotherm are very similar to the results for silica gel alone. As for wettability tests results, the quantity of MgSt (1\%) used was not sufficient to reduce the uptake of water on silica gel after coating.

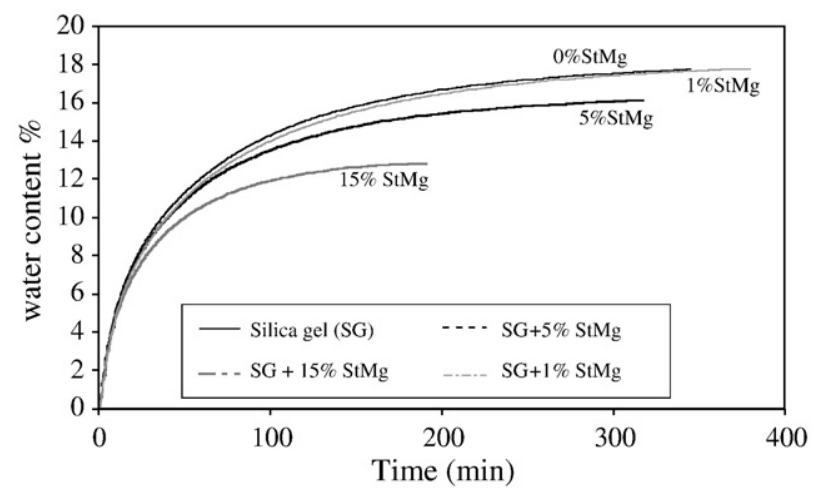

Fig. 9. Kinetics of moisture adsorption at $25^{\circ} \mathrm{C}$, silica gel and silica gel coated with MgSt.

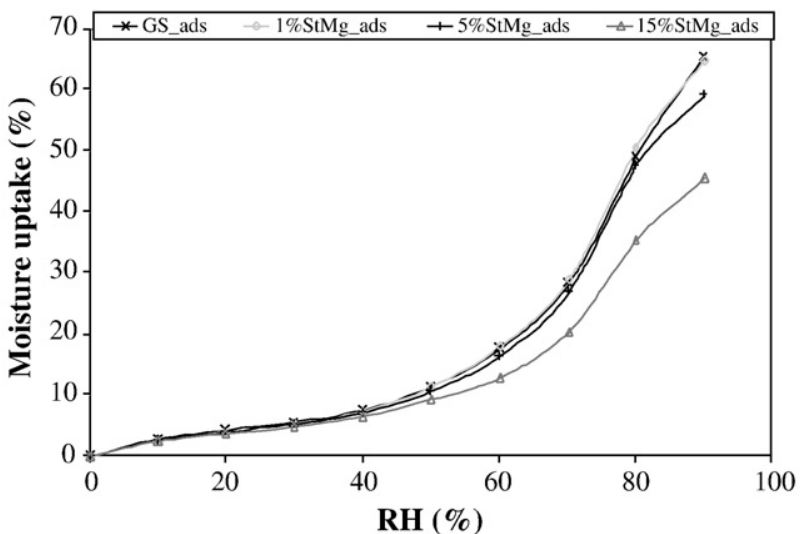

Fig. 10. Moisture adsorption isotherm $25{ }^{\circ} \mathrm{C}$, silica gel and silica gel coated with MgSt. 


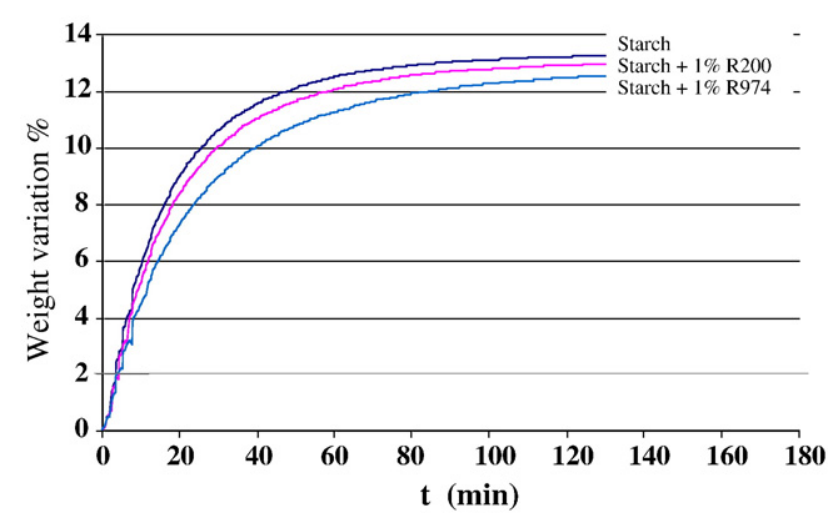

Fig. 11. Moisture adsorption kinetics for a step change from 0 to $60 \%$ relative humidity.

The total amount of the water sorbed decreased by almost $10 \%$ for silica gel particles coated with 5\% of MgSt and 30\% for particles coated with $15 \%$ of MgSt. This suggests that the $5 \%$ and $15 \% \mathrm{MgSt}$ coating impermeabilise the silica gel surface and may be in the form of a continuous film.

\subsubsection{Starch, fume silica system}

Fig. 11 shows the kinetics of moisture absorption of starch and starch coated with R200 (hydrophilic) and R974 (hydrophobic) fume silica. It can be seen that the silica coatings have a slight slowing down effect of moisture uptake and that the hydrophobic coating slows uptake more than the hydrophilic coating. However the final plateau value reached is practically the same in each case indicating that overall amount of moisture taken up is the same. This means that the absorption isotherm is unchanged by the silica coatings. This is in contrast to the results with MgSt coatings given above, and could suggest that the fume silica is in the form of isolated individual particles and does not prevent moisture reaching the starch.

\subsection{Tribo-electric charging}

\subsubsection{Silica gel magnesium stearate system}

Fig. 12 shows the tribo-charging characteristics for the silica gel alone and coated with $1 \%, 5 \%$, and $15 \% \mathrm{MgSt}$. It can be seen that the uncoated silica gel has an initial negative charge of about $1.5 \mu \mathrm{C}$ per gram and which remains over the test period of $30 \mathrm{~min}$ rotation in the stainless steel beaker. The silica gel samples coated with MgSt have a positive charge of around $0.5 \mu \mathrm{C}$ per gram and this remains constant over the experiment. There is no substantial difference in the results for the

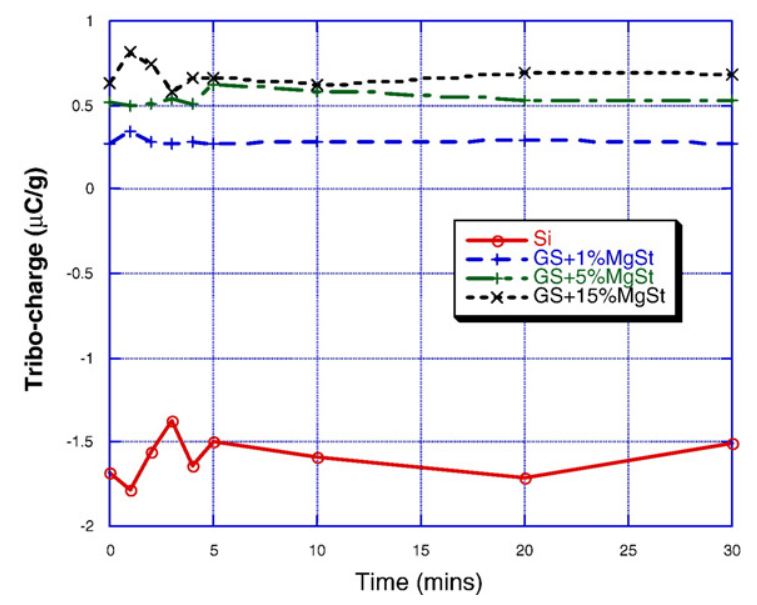

Fig. 12. Tribo-charging characteristics for silica gel and silica gel coated with MgSt.

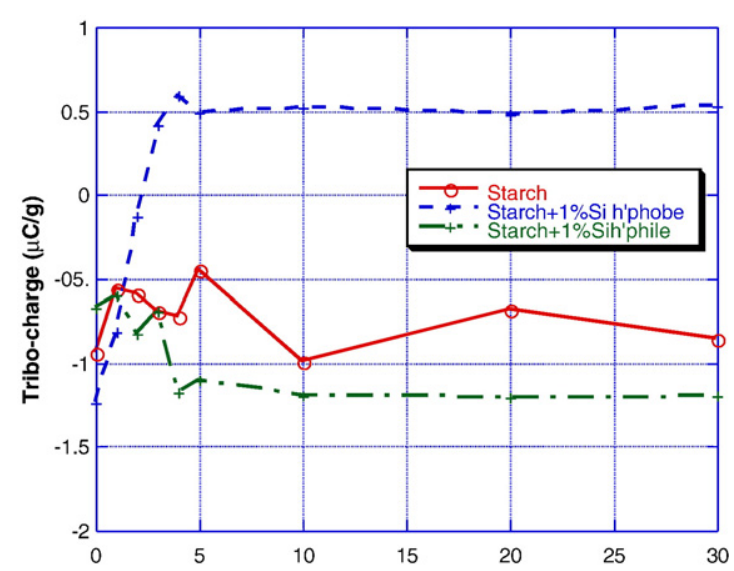

Fig. 13. Tribo-charging characteristics for starch and starch coated with fume silica.

different coatings except that for the $1 \%$ coating is lower than the other two, and the $15 \%$ seems to be slightly higher than the results for the $5 \%$ coating.

\subsubsection{Starch, fume silica system}

Fig. 13 shows the tribo-charging characteristics of the untreated starch and starch coated with R200 (hydrophilic) and R974 (hydrophobic) fume silica. It can be seen that the starch by itself has a negative charge of around $0.8 \mu \mathrm{C}$ per gram, which remains stable over the duration of the experiment. The results for the starch coated with hydrophilic fume silica are similar with a negative charge of about $1 \mu \mathrm{C}$ per gram. The results for the starch with the hydrophobic coating are very different. The initial charge is similar to the others at a value of about $-1 \mu \mathrm{C}$ per gram. This rises rapidly over the first $5 \mathrm{~min}$ to become positive at a value of about $0.5 \mu \mathrm{C}$ per gram.

\section{Conclusions}

This study indicates the Cyclomix can be used for dry powder coating of particles and that the mechanical resistance of the coatings is good, as determined by a laser diffraction particle size dispersion test. Two powder-powder systems have been tested: silica gel particles coated with hydrophobic magnesium stearate and starch particles coated with either hydrophilic or hydrophobic fume silica. The silica gel treated alone in the Cyclomix was slightly fragmented and the starch was relatively undamaged. The ESEM images of the uncoated and coated particles showed that MgSt seemed to be smeared over host particles and this effect increased as the MgSt mass ratio increased to 15\%. The two types of fume silica were fixed on the surface as individual particles.

The changes in overall powder characteristics have been determined by tap-tap test for cohesion and flowability using the Carr and Hausner indices, the wettability by sessile drop and DVS measurements and by tribo-charging tests. The flowability of the silica gel powder was significantly decreased after treatment the Cyclomix mixer with $15 \%$ of MgSt. It is also found that the coating by hydrophobic MgSt in the Cyclomix can also reduce the high affinity between silica gel and water after treatment with $5 \%$ and $15 \%$ of MgSt. The tribo-charging tests show that coating the Silica gel with MgSt changes them from negative to positively charged particles. The settling characteristics of the starch particles was significantly changed by coating with fume silicas giving high bulk densities. However the Carr and Hausner indices were found not to be able to discriminate. The overall moisture adsorption isotherm was little changed by the coating but the kinetics of moisture uptake was found to be significantly slower for the coated starch particles. Finally the tribo-charging tests show a significant different in the case of the hydrophobic coating. To summarize, it has been demonstrated that the high shear 
mixer "Cyclomix" can be used as dry coating device to change the properties of host powders.

\section{References}

[1] I. Yoshihara, W. Pieper, Hybridization - technology for surface modification of powders without binders, Swiss Pharma 21 (1999) 6.

[2] K. Saleh, P. Guigon, Chapter 7: Coating and encapsulation processes in powder technology, Handbook of Powder Technology, vol. 11, 2007, pp. 323-375.

[3] R. Pfeffer, R.N. Dave, W. Dongguang, M. Ramlakhan, Synthesis of engineered particulates with tailored properties using dry particle coating, Powder Technology 117 (2001) 40-67.

[4] S. Kangwantrakool, K. Shinohara, Preparation of new WC-Co/TiC- $\mathrm{AL}_{2} \mathrm{O}_{3}$ composite materials with mechanically coated particles, Journal of Chemical Engineering of Japan 34 (2001) 1486-1492.

[5] J.A. Hersey, Ordered mixing: a new concept in powder mixing, Powder Technology 11 (1975) 41-44.

[6] H. Honda, K. Ono, T. Ishizaka, T. Matsuno, M. Katano, Surface modification of powders by the high speed impact treatment method, Journal of the Society of Powder Technology Japan 24 (1987) 593-599.

[7] H. Honda, T. Matsuno, M. Koishi, Preparation of a graphite fluoride modifiedpolymer microsphere by a high speed impact treatment method, Journal of the Society of Powder Technology Japan 25 (1988) 597-602.

[8] H. Honda, T. Matsuno, M. Koishi, The effect of powder properties on dry impact blending preparation method, Journal of the Society of Powder Technology Japan 25 (1989) 666-671.
[9] H. Honda, M. Kimura, F. Honda, T. Matsuno, M. Koishi, Preparation of composite and encapsulated powder particles by dry impact blending, International Journal of Chemistry and Biotechnology 9 (1991) 21.

[10] A. Mujumdar, D. Wei, R.N. Dave, R. Pfeffer, C.Y. Wu, Improvement of humidity resistance of magnesium powder using dry particle coating, Powder Technology 140 (2004) 86-97.

[11] J. Yang, A. Sliva, A. Banerjee, R.N. Dave, R. Pfeffer, Dry particle coating for improving the flowability of cohesive powders, Powder Technology 158 (2005) 21-33.

[12] B.H. Ng C.C. Kwan, Y.L. Ding, M. Ghadiri, X.F. Fan, Solids motion in a conica frustum-shaped high shear mixer granulator, Chemical Engineering Science 62 (2007) 756-765.

[13] C.C. Kwan, B.H. Ng, Y.L. Ding, R.A. Williams, M. Ghadiri, X.F. Fan, D.J. Parker, Effect of macroscopic flow field on the evolved structure and properties of granules during scaling-up of high shear granulators, 8th International Symposium on agglomeration, Thailand, 2005

[14] A. Vilela, L. Concepcion, P. Accart, A. Chamayou, M. Baron, J.A. Dodds, Evaluation of the mechanical resistance of a powder-powder coating by modulated dry feed particle size analysis, Particle \& Particle Systems Characterization 23 (2006) 127-132.

[15] M. Lazghab, K. Saleh, I. Pezron, P. Guigon, L. Komunjer, Wettability assessment of finely divided solids, Powder Technology 157 (2005) 79-91.

[16] C. Goalard, L. Galet, J.A. Dodds, Characterization of the dispersion behavior of talc, $8^{\text {th }}$ International Symposium on Agglomeration, Proceedings ed N. Sinchaipanid \& al. pp. 121-128, ISBN 974-9717-76-7, Characterisation and Measurement Techniques, Bangkok, Thailand, 16-18 March (2005).

[17] C. Gatumel, M. Lallée, J. Revel, Etude expérimentale de la charge par frottement de particules de polyéthylène, Récents Progrès en Génie des Procédés, vol 91, CD-ROM édition., 4th Colloque "Science et Technologie des Poudres" Compiegne, 4-6 Mai 2004. 